\title{
Modelling with the 2D Multifluid Code TECXY of TEXTOR Discharges in the Presence of DED
}

\author{
H.Gerhauser ${ }^{1}$, R.Zagórski ${ }^{2}$, G.Telesca ${ }^{3}$ \\ ${ }^{1}$ Institut für Plasmaphysik, Forschungszentrum Jülich GmbH, \\ EURATOM Association, D-52425 Jülich, Germany \\ ${ }^{2}$ Institute of Plasma Physics and Laser Microfusion, \\ EURATOM Association, P.O.Box 49, 00-908 Warsaw, Poland \\ ${ }^{3}$ Department of Applied Physics, Ghent University \\ Rozier 44, B-9000 Gent, Belgium
}

\begin{abstract}
A study of the influence of the Dynamic Ergodic Divertor (DED) on transport and radiation of intrinsic carbon in TEXTOR has been done, based on calculations with the 2D multifluid code TECXY and experimental data from spectroscopic diagnostics involving the brilliances of lines emitted by $\mathrm{C}^{+2}$ and $\mathrm{C}^{+4}$ lines in the $\mathrm{UV}$ range. This allows to investigate the radiation properties of carbon and the contamination of the main plasma for a variety of experimental conditions. The physical model in the TECXY code was extended to incorporate the increased radial transport in the stochastic magnetic field by applying a model of "optimal paths". Two DED operational modes $(m / n=12 / 4$ and $3 / 1)$, both resonant at the $\mathrm{q}=3$ surface, have been experimentally studied so far. In the present paper we have concentrated on the 12/4 mode of operation. The radial decay length of the 12/4 mode is very short. In order to maximize the field penetration, the plasma column has to be shifted horizontally towards the HFS. Both the simulation and the experimental evidence indicate that the main changes are related to the horizontal shift of the plasma column rather than to the stochastic transport itself. A high recycling regime with good impurity screening is predicted for a shifted position of the plasma column.
\end{abstract}

\section{Introduction}

One of the most critical issues on the way to the tokamak-reactor is the problem of the heat and particle exhaust. A possible solution might involve the creation of an ergodic layer in the plasma boundary region. Such an idea has been implemented on TEXTOR tokamak by installing the Dynamic Ergodic Divertor (DED) with the aim of producing a perturbed magnetic field resonant at the $\mathrm{q}=3$ surface [1]. The study of the response of edge plasma parameters and in particular of the intrinsic carbon to a change in the edge magnetic field structure can provide a key element to assess the viability of the DED concept to reactor grade experiments. Such a study has been done using both the 2D multifluid code TECXY and spectroscopic measurements.

The physical model in the TECXY code is based on Braginskij-like equations for the background plasma and rate equations for the impurity ions [2]. The code incorporates drift motions and currents in a fully self-consistent way. The increased effective radial transport coefficients in the stochastic magnetic field are calculated according to Tokar's [3] model of optimal paths, 
which leads to analytical formulae involving the fieldline diffusion coefficient $D_{F l}$ and the Kolmogorov length $L_{K}$ as the essential parameters.

The brilliances of the $\mathrm{C}^{+2}$ ion line and of the $\mathrm{C}^{+4}$ line in the UV range are detected simultaneously along a set of lines of sight distributed over a significant fraction of the poloidal angle near the High Field Side (HFS) where the coils of the ergodic divertor are located. This allows, together with the measurements of the total radiated power and $Z_{\text {eff }}$, to investigate the radiation properties of carbon and the contamination of the main plasma for a variety of experimental conditions. Our modelling investigations with TECXY code have been performed in order to identify the main physical mechanisms responsible for the observed changes of the edge parameters.

Two DED operational modes $(m / n=12 / 4$ and $3 / 1)$, both resonant at the $\mathrm{q}=3$ surface, have been experimentally studied so far. In contrast to modes with lower $m$ the radial decay length of the $12 / 4$ mode is very short. In order to maximize the field penetration, the plasma column has to be shifted horizontally towards the HFS. This implies a change on the location of recycling and of impurity source (the main plasma sink at the ALT-II limiter being replaced by the sink at the DED surface), which affects the poloidal asymmetries in the plasma edge. In the present paper we have considered only the $12 / 4$ mode of operation since in the $3 / 1$ mode of operation large islands are created in the central part of the plasma which can not be treated in the frame of the 2D boundary layer code.

Results of calculations and experiment indicate that the effect of plasma shift might be of major importance. For different plasma configurations the effect of the ergodic layer has been investigated in detail. The paper is organized in the following way. The physical model and the description of the stochastic transport are outlined in Sec.2. In Sec.3 the diagnostic set-up is presented together with experimental results. The results of numerical simulations are shown and discussed in section 4 , followed by conclusions.

\section{Physical model}

We use an updated version of the 2D multifluid code TECXY [2],[4]-[8] to model possible changes of edge plasma transport as produced by the dynamic ergodic divertor (DED) [9], which has been installed at the high-field side of TEXTOR tokamak. The DED creates a perturbation field which generates a stochastic (ergodic) boundary layer in the outermost region of the plasma near the separatrix. The ergodicity is intended to enhance the radial diffusive and convective transport significantly. The DED replaces the former bumper limiter which was liable to trigger the evolution of Marfes. Since the parallel and radial transport of particles and heat is of crucial importance for the onset and strength of Marfes, the stochastic magnetic field, as produced by the magnetic perturbations from DED coils, affects strongly the Marfe evolution [10, 11]. In the present paper we will restrict on experimental conditions and parameter regimes where the onset of Marfes can be avoided. 
The most important code extensions concern the correct formulation of the anomalous transport in the electron/ion energy balance [12] and the incorporation of anomalous shear viscosity effects in the ion momentum balance, which allows to fulfil the global (magnetic surface averaged) ambipolarity constraint for the radial electric current, and to determine the electric field in the transition layer by a second order ordinary differential equation for $E_{r}(r)$ [13].

Over the last few years the physical model implemented in the TECXY code was applied to investigate different experimental situations. In particular the code was successfully used to explain the biassing and polarization experiments on TEXTOR tokamak $[2,14]$. It was possible with the code results to explain the MARFE appearance and evolution in TEXTOR tokamak plasmas $[5,10,11]$ as well as the observed poloidal asymmetries of plasma parameters in the edge[15].

\subsection{Basic features implemented in the code}

The TECXY code solves Braginskij-like equations for the background plasma and rate equations for the impurity ions. The model describes the electrons and various ion species in their different charge states as separate fluids. The transport along field lines is assumed to be classical, and the radial transport is anomalous with prescribed radial transport coefficients of the order of Bohm diffusion and Alcator-like radial dependence. An analytical description of two groups of neutrals allows to take into account plasma recycling as well as sputtering and self-sputtering of impurity atoms at the ALT-II limiter surface. The present version of the code ([2], [13]) incorporates drift motions and currents in a fully self-consistent way with plasma and impurity dynamics. In order to consider plasma drifts and currents, additional equations are added to standard equations, which are obtained from the diamagnetic and radial components of Ohm's law and the equations of motion. Thus we consider in the model the perpendicular drift velocities and, in addition to the radial diffusion velocities, also radial drift velocities $V_{r}^{\text {drift }}$. The radial electric field and plasma potential in the SOL (Scrape-off Layer) are determined from the parallel component of the Ohm's law, whereas in in the transition layer inside the separatrix they are derived from an ordinary differential equation which ensures global ambipolarity of the radial electric current. The details of the model are described in [2],[4]-[8], [13] and the references cited therein. In this paper we concentrate on the role of neutrals recycled from the DED surface and on the role of stochastic transport from the DED perturbation field for plasma and carbon transport, radiation and carbon screening. The geometry of the boundary layer is sketched in Fig.1 for normal polarity (orientation) of the plasma current $I_{p}$ and the main (toroidal) magnetic field $B_{\phi}$ of TEXTOR tokamak. For every ion species we solve the continuity, parallel momentum and energy equations. All ion species are assumed to have the same common temperature $T_{i}$, which differs from the electron temperature $T_{e}$.

In order to model the DED region (which replaces the former bumper region) we have assumed high additional recombination losses inside this region and a suitably chosen profile for the recycled neutrals penetrating from the DED surface into the adjacent plasma. For this 


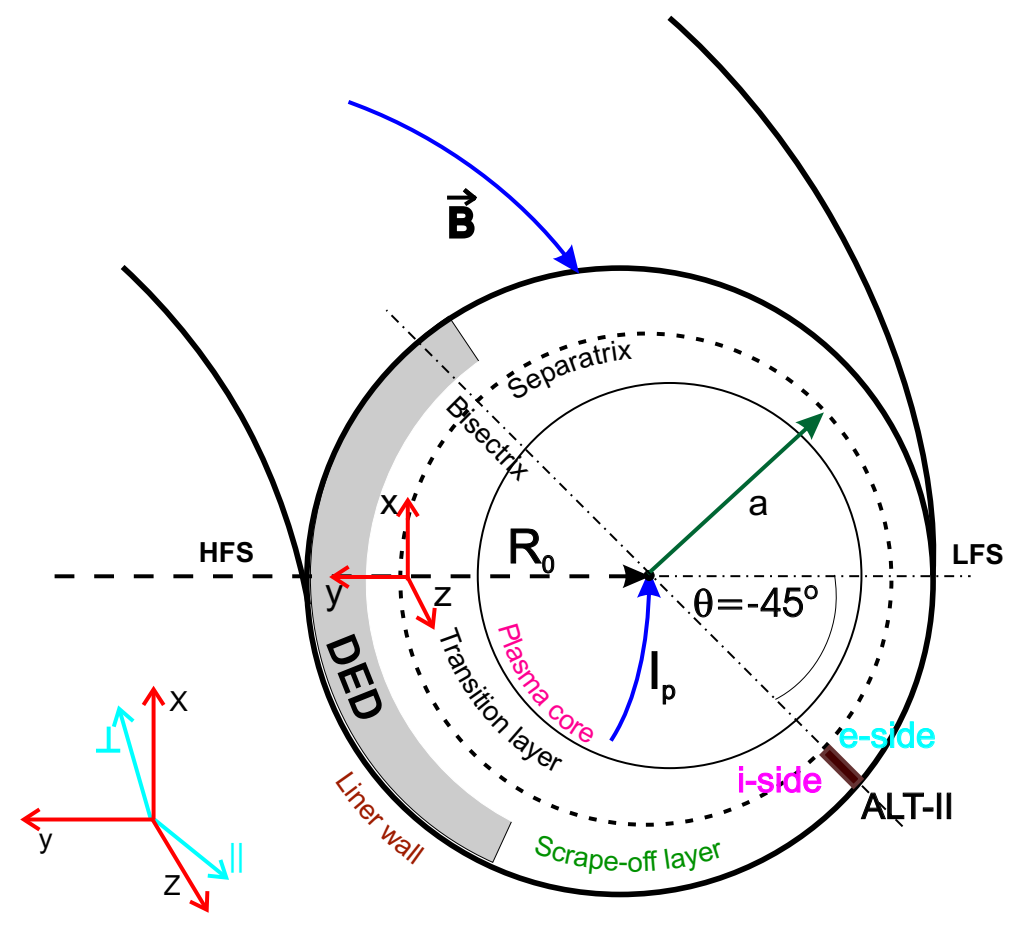

Figure 1: Boundary layer geometry (schematic) for TEXTOR tokamak with toroidal belt limiter and Dynamic Ergodic Divertor. The poloidal angle $\theta$ changes from $0^{\circ}$ at Low Field Side (LFS) to $\pm 180^{\circ}$ at High Field Side (HFS). The ALT-II limiter is at the $\theta=-45^{\circ}$ position, whereas the DED covers in reality the region $120^{\circ}<\theta<240^{\circ}$. The bisectrix is defined as a symmetry line crossing the target.

additional group of deuterium neutrals arising at the DED the profile $N_{D}^{\text {ded }}(x, y)$ is prescribed by a product of exponential functions, involving the mean free path $\lambda_{D}$ of neutrals released from the DED. The proper normalization of the deuterium neutral density in front of the DED is determined by the losses in the DED region and by the requirement of complete reionization of the recycled neutrals $\left(\alpha_{i}^{D}\right.$ and $\alpha_{c x}^{D}$ are rate coefficients for ionization and charge exchange):

$$
\int_{V O L} N_{D}^{d e d}(x, y) \alpha_{i}^{D}(x, y) n_{e}(x, y) d V=\int_{V O L} n_{e}(x, y) \beta_{l o s s}(x, y) n_{i}(x, y) d V
$$

where

$n_{e}, n_{i}$ are electron and ion densities respectively and $V O L$ is the volume of the boundary layer, $\beta_{\text {loss }}$ is the assumed recombination rate coefficient equal to $2 \times 10^{-14} \mathrm{~m}^{3} \mathrm{~s}^{-1}$ in the DED region and equal to 0 outside the DED. The exact value of the volume recombination $\beta_{\text {loss }}$ is not important if it is high enough to guarantee fast and perfect recombination of the incident plasma flux. The temperature of deuterium atoms is calculated from the equilibrium condition in the region in front of the DED surface $T_{D}^{d e d}=\alpha_{c x}^{D} T_{i} /\left(\alpha_{i}^{D}+\alpha_{c x}^{D}\right)$ and is assumed to be constant outside this region: $T_{D}^{\text {ded }}=T_{D o}^{\text {ded }}=0.02-0.05 \mathrm{eV}$. It is assumed in the model that the recycled deuterium atoms travel radially with the thermal velocity. For impurity atoms (carbon) a similar model has been used. The temperature of impurity atoms is taken to be $T_{C}^{d e d}=3.5 \mathrm{eV}$, and they travel radially with thermal velocity. For carbon atoms we have assumed additionally that apart from the neutrals recycling at the DED we have also an influx from the DED $\left(\Gamma_{\text {ded }}^{C}\right)$ due to sputtering processes (physical and chemical). For simplicity we have taken $\Gamma_{d e d}^{C}=Y_{D} \Gamma_{d e d}^{D}$, 
where $\Gamma_{\text {ded }}^{D}$ is the plasma flux to the DED. The sputtering yield $Y_{D}$ is a sensitive function of the plasma temperature in front of the DED. It is equal to about 0.01 and limited to a maximum value of 0.025 .

\subsection{Transport in stochastic fields}

It is hoped that the artificial stochastization at the plasma edge due to the operation of the DED is an effective tool to control transport and to optimize plasma-wall interaction in TEXTOR. In the following we give some explanations and background to Tokar's [3] transport formulae. The transport coefficients in a stochastic magnetic field are computed by taking into account analytical relationships for the distribution of plasma parameters along divergent lines of force. The transport in a stochastic field is considered as a sequence of displacements both parallel and perpendicular to the magnetic field lines, which are linked together forming "optimal paths" with largest effective radial transport. Due to the stochasticity of the magnetic field, the radial distance between parallel sections of the path changes with their length $l$. For small $l$ this distance obeys the exponential law $\delta_{1}=\delta(l)=\delta_{0} \exp \left(l / L_{K}\right)$ and characterizes the local divergence of field lines in the stochastic layer, where $L_{K}$ is the Kolmogorov length. For large $l$ this is replaced by a diffusive law $\delta_{1}=\delta(l)=\sqrt{2 D_{F l} l}$ where $D_{F l}$ is the field line diffusivity and has also the dimension of a length $\left[\mathrm{m}^{2} / \mathrm{m}\right]$. Both parameters are determined by the spectrum and strength of the field perturbations and can be varied independently.

For the heat conductivity of either electrons or ions (with the letter $\chi$ for heat conductivities) the maximum radial effective heat conductivity is found to be

$$
\chi_{\perp}^{\text {path }}=\chi_{\perp} \frac{1+\zeta_{T} x e^{-x}}{1+\zeta_{T} x e^{-2 x}} \quad \text { with } \quad x=l / L_{K} \quad \text { and } \quad \zeta_{T}=\left(2 D_{F l} / L_{K}\right) \chi_{\|} / \chi_{\perp} .
$$

where $\chi_{\|}$is the parallel (along magnetic field lines) heat conductivity. $\chi_{\perp}^{\text {path }} / \chi_{\perp}$ has a maximum with respect to $x$, say at $x=x_{T}$, which yields an effective $\chi_{\perp}^{e f f} / \chi_{\perp}$ and is plotted in Fig.2a as a function $\psi_{T}\left(\zeta_{T}\right)$. Hence we use in the code

$$
\chi_{\perp}^{e f f}=\chi_{\perp}+\chi_{\perp}^{D E D}=\chi_{\perp}+\chi_{\perp}\left(\psi_{T}\left(\zeta_{T}\right)-1\right) .
$$

where $\chi_{\perp}$ is the radial (anomalous) heat conductivity. For the ions the influence of stochasticity on the effective heat conductivity is much weaker because of the much lower parallel heat conductivity of ions in comparison to that of electrons. On the other hand $\chi_{\perp}$ is also smaller for ions, and in view of the temperature dependence of $\chi_{\|}$like $T^{5 / 2}$ a higher ion temperature largely increases $\chi_{\|} / \chi_{\perp}$ for the ions. Hence even for ions the effect of stochasticity may be locally non negligible.

For the transport of background ions along a path of dimensions $\delta_{0}$ and $l$ it is assumed that there is no intrinsic perpendicular pinch velocity besides the anomalous diffusion, and that the parallel pressure gradient is balanced by the perpendicular transfer of parallel momentum with the anomalous viscosity $m_{i} n \mu_{\perp} \approx m_{i} n D_{\perp}$ and is estimated as

$$
\partial P / \partial l=\partial / \partial l\left(n T_{e}+n T_{i}\right)=-m_{i} n \mu_{\perp} V_{\|} / \delta^{2} .
$$



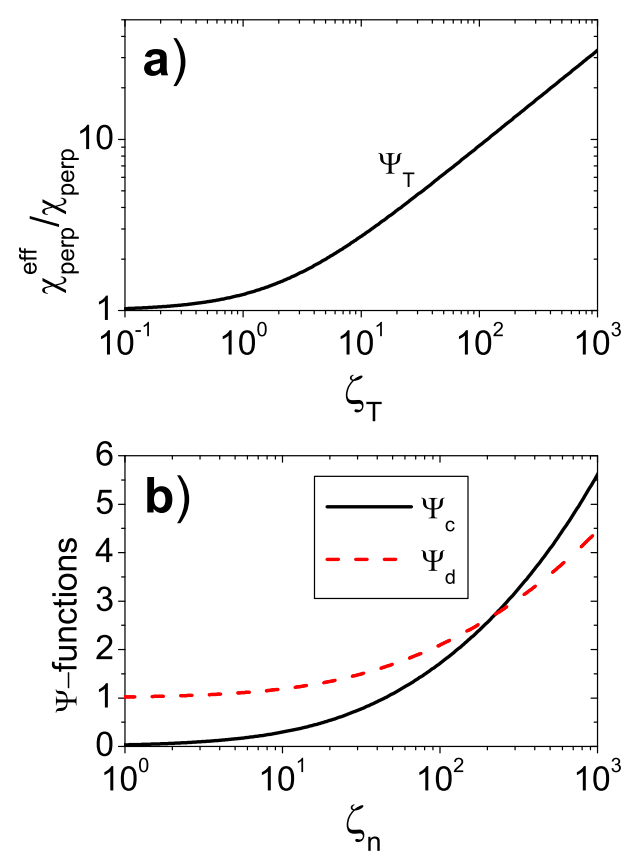

Figure 2: Stochastically increased heat conduction (a) and ion convection/diffusion (b). $\Psi_{d}$ stands for the diffusive part of the radial particle transport, whereas $\Psi_{c}$ corresponds to the convective part of the particle flux.

where $m_{i}$ is the ion mass, $n=n_{e}=n_{i}$ stands for plasma density, $\mu_{\perp}, D_{\perp}$ are the anomalous viscosity and diffusion coefficients, respectively. $P$ stands for the total plasma pressure and $V_{\|}$is the parallel plasma velocity. The final radial flux $\Gamma_{r}$ is composed of a diffusive and a convective (pinch) part:

$$
\begin{aligned}
\Gamma_{r} & =-D_{\perp}^{\text {path }} \nabla_{r} n+V_{r}^{\text {path }} n \\
\text { with } \quad D_{\perp}^{\text {path }} & =D_{\perp} \frac{1+g(x)}{1+g(x) e^{-x}}, \quad V_{r}^{\text {path }}=\frac{D_{\perp}}{L_{T}^{\text {mod }}} \frac{g(x) \sqrt{x}}{1+g(x) e^{-x}} \\
\text { where } g(x) & =\zeta_{n} x^{2} \frac{1-e^{-x}}{e^{3 x}-1}, \quad \zeta_{n}=12 \frac{\left(D_{F l} c_{s}\right)^{2}}{D_{\perp} \mu_{\perp}} \\
\text { and } \quad L_{T}^{\text {mod }} & =\frac{T_{e}+T_{i}}{-\nabla_{r}\left(T_{e}+T_{i}\right)} \frac{\sqrt{x_{T}}}{1-e^{-x_{T}}}
\end{aligned}
$$

with the above mentioned $x_{T}$ for optimal paths of heat transport. The maxima of $D_{\perp}^{\text {path }}$ and $V_{r}^{\text {path }}$ occur at about the same $x=x_{n}$ (which is different from $x_{T}$ ), yielding

$$
\Gamma_{r}=-D_{\perp}^{e f f} \nabla_{r} n+V_{r}^{\text {eff }} n=-D_{\perp} \psi_{d}\left(\zeta_{n}\right) \nabla_{r} n+\left(V_{r}^{\text {drift }}+V_{r}^{D E D}\right) n .
$$

The $\psi$-functions are plotted in Fig.2b and correspond to the aforementioned maxima of $D_{\perp}^{\text {path }}$ and $V_{r}^{\text {path }}$. Hence we use in the code

$$
D_{\perp}^{e f f}=D_{\perp}+D_{\perp}^{D E D}=D_{\perp}+D_{\perp}\left(\psi_{d}\left(\zeta_{n}\right)-1\right), \quad \text { and } \quad V_{r}^{D E D}=\left(D_{\perp} / L_{T}^{\text {mod }}\right) \psi_{c}\left(\zeta_{n}\right)
$$

the usual radial pinch velocity being supplemented by an additional effective radial drift from the stochasticity in the DED field. It is important to note that $\zeta_{n}$ involves only $D_{F L}$, but not $L_{K}$. 
For the calculation of impurity diffusion in the stochastic field it is assumed that trace impurities move in a homogeneous background plasma at rest. The impurity parallel velocity $V_{\| z}$ is determined also by the friction with the main ions:

$$
T_{i} \partial n_{z} / \partial l=-m_{z} n_{z} \mu_{\perp} V_{\| z} / \delta^{2}-m_{i} m_{z} /\left(m_{i}+m_{z}\right) \nu_{i z} n_{z} V_{\| z}
$$

where $\nu_{i z}$ is the collision frequency of the impurity ions with the background ions and $m_{z}$ is the mass of impurity ions. This leads to an effective impurity diffusion coefficient

$$
\begin{gathered}
D_{\perp z}^{e f f}=D_{\perp} \psi_{d z}\left(\zeta_{z}, \xi_{z}\right)=D_{\perp}+D_{\perp z}^{D E D}=D_{\perp}+D_{\perp}\left(\psi_{d z}\left(\zeta_{z}, \xi_{z}\right)-1\right) \\
\text { with } \zeta_{z}=\frac{12 D_{F l}^{2} T_{i}}{D_{\perp} \mu_{\perp} m_{z}} \quad \text { and } \quad \xi_{z}=\frac{6 D_{F l} L_{K} \nu_{i z}}{\mu_{\perp}\left(1+m_{z} / m_{i}\right)} .
\end{gathered}
$$

However, the additional diffusion $D_{\perp z}^{D E D}$ from stochasticity corresponding to the "optimal" paths is usually very small.

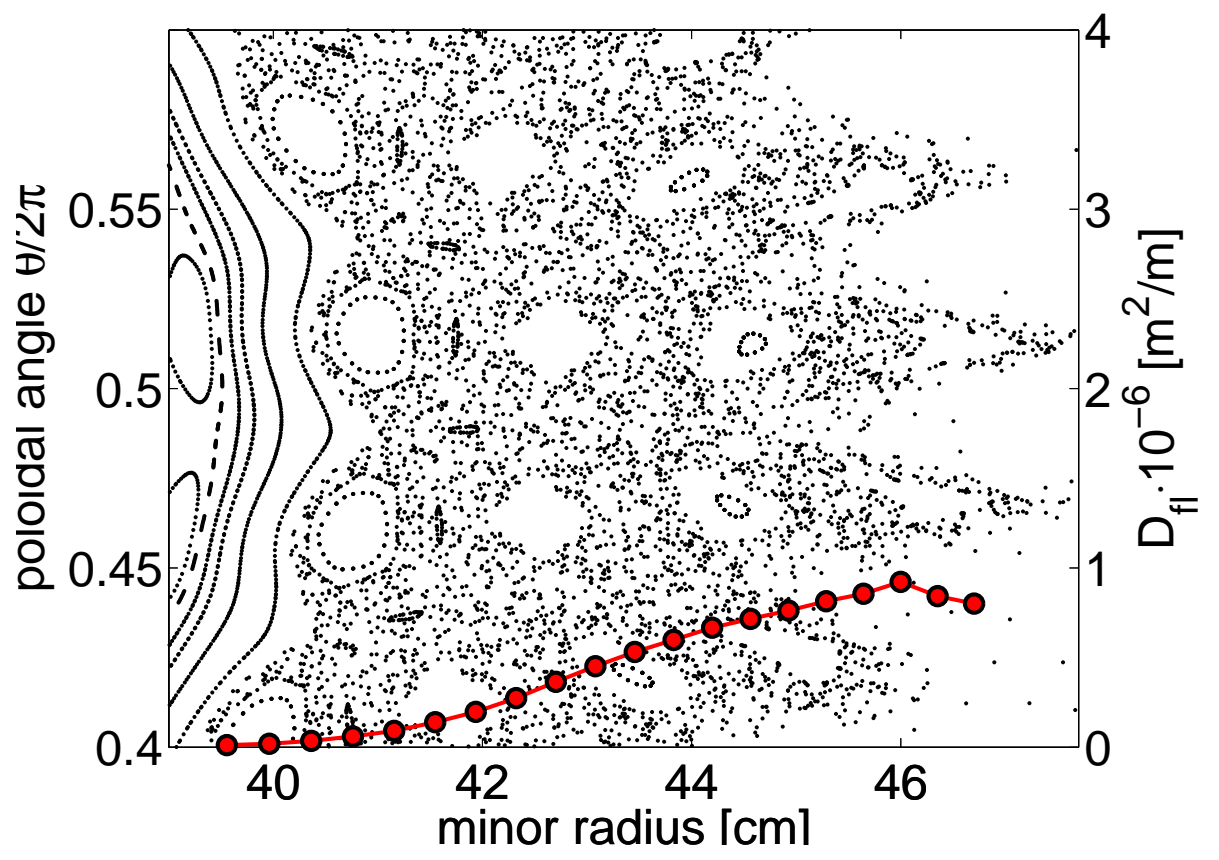

Figure 3: Poincaré section of perturbed field lines at the plane $(\theta, y)$ [16]. The plasma parameters correspond to a typical TEXTOR discharge: $\beta_{p o l}=0.3$, plasma current $I_{p}=280 \mathrm{kA}$, DED current $I_{D E D}=11 \mathrm{kA}$, toroidal field $B_{T}=1.9 \mathrm{~T}$, major radius of the magnetic axis $R=168 \mathrm{~cm}$ (shifted plasma). The profile of the field diffusion coefficient $D_{F L}$ is also shown.

Since a realistic plasma is inhomogeneous and has flow velocities, the impurities will be influenced by Coulomb collisions and acquire an additional convective motion. We consider the parallel section of an optimal path for the transport of background particles and find that the mass velocity $U_{\| z}$ of impurity ions is governed by the equation

$$
m_{z} U_{\| z} \partial U_{\| z} / \partial l=m_{i} m_{z} /\left(m_{i}+m_{z}\right) \nu_{i z}\left(V_{\|}-U_{\| z}\right)+\alpha_{T}^{e z} \partial T_{e} / \partial l+Z e E_{\|}
$$

with the friction force from the background ions, the thermal force $\left(\alpha_{T}^{e z}=0.71 Z^{2}, Z e\right.$ is the impurity ion charge) and the electric force, the latter being determined from the parallel Ohm's 
law. Integrating and averaging along the optimal path yields with some approximations a quadratic equation for the characteristic parallel velocity $\overline{U_{\| z}}$, which gives a contribution $V_{r z}^{D E D}$ to the radial impurity convection:

$$
V_{r z}^{e f f}=V_{r z}^{d r i f t}+V_{r z}^{D E D}=V_{r z}^{d r i f t}+\overline{U_{\| z}} \sqrt{2 D_{F l} / L_{K}}\left(1-\exp \left(-x_{n}\right)\right)^{2} / \sqrt{x_{n}}
$$

which is governed mainly by friction with the background ions and thus directed radially outward. It may become quite large and thus help to screen the plasma core from impurities, which decreases the effective charge, $Z_{\text {eff }}$, in the plasma core.

The above presented transport model has been implemented into the edge code TECXY to perform a numerical modelling of the influence of DED on plasma parameters in the boundary region of TEXTOR tokamak. The values we use for the fieldline diffusion coefficient $D_{F L}$ and the Kolmogorov length $L_{K}$ are derived from numerical reconstruction of the magnetic structure at the plasma edge. In Fig.3 we show a Poincaré section of field lines at the plasma boundary together with the profile of the field diffusion coefficient $D_{F L}$ for a typical TEXTOR-DED discharge. The island chain corresponding to the main resonant surface with a width of about 1 $\mathrm{cm}($ at $q=3)$ is clearly visible at $y \approx 44 \mathrm{~cm}$. Closer to the core one can recognize other resonances corresponding to $\mathrm{m}=11$ and $\mathrm{m}=10$, while near the target a fingers structure develops. (We note that in the frame of the present model we cannot treat the transition to laminar transport in a zone very close to the DED surface.)
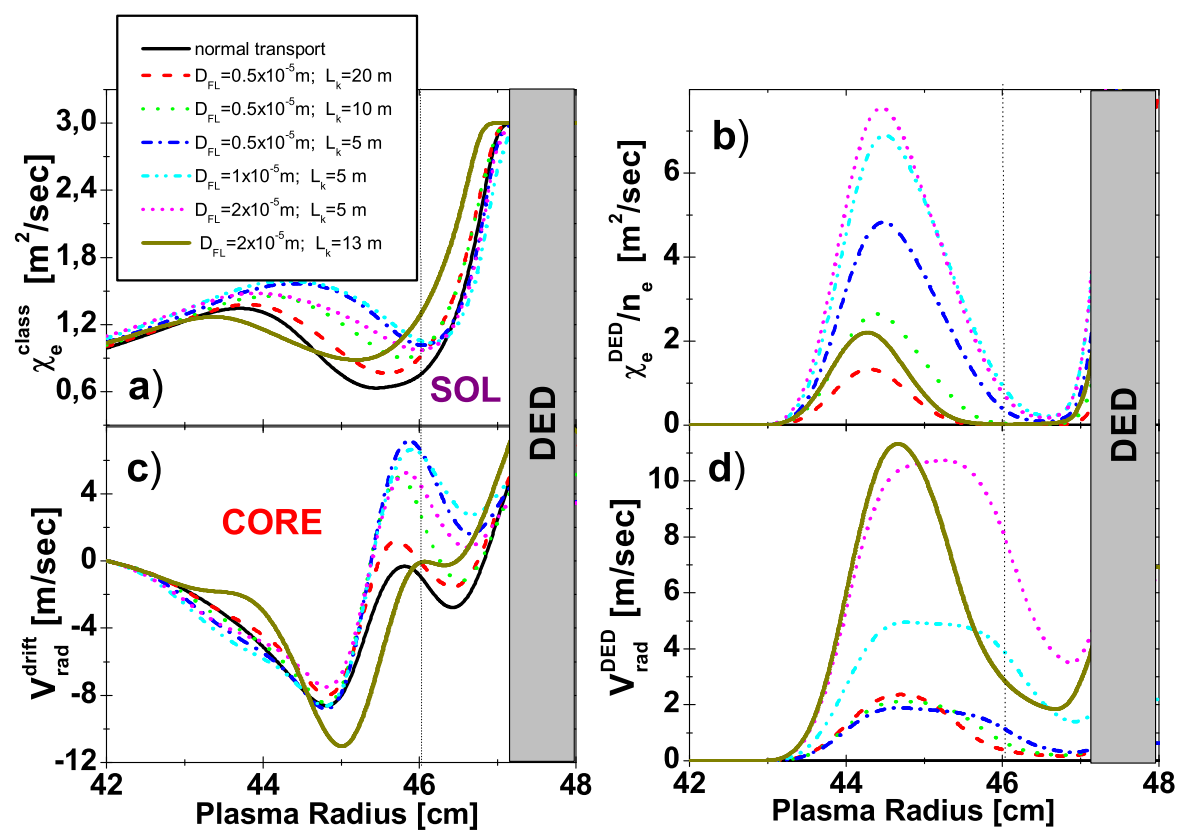

Figure 4: Radial profiles of the heat conductivity and particle convection. Calculations are performed for different values of $D_{F L}$ and $L_{K}$. The thin vertical line marks the position of the plasma separatrix.

Following refs. $[9,17,18]$ typical values in the ergodic zone in front of the DED surface near 
the separatrix are $D_{F l}=0.4 \times 10^{-5} \mathrm{~m}^{2} / \mathrm{m}$ and $L_{K}=20 \mathrm{~m}$ or larger depending on the plasma current and the magnitude and distribution of the helical perturbation currents in the DED coils. Since there is a fast radial decay of $D_{F l}$ and radial increase of $L_{K}$ beyond $y=43 \mathrm{~cm}$ towards the plasma core, the stochastic transport coefficients are reduced to zero in the code between $y=43.5 \mathrm{~cm}$ and $y=42.5 \mathrm{~cm}$ by an s-like function, and they are poloidally restricted to the DED-region, that is the sector $120^{\circ}<\theta<240^{\circ}$. Radially and poloidally far away from the DED-coils the induced magnetic field perturbations are so small that the plasma particles see only the unperturbed TEXTOR field and have the standard transport coefficients without stochastic corrections. Some aspects of the influence of the stochasticity parameters on the plasma transport have already been analyzed in ref. [8] for a wide range of the stochasticity parameters: $0.5 \times 10^{-5} \mathrm{~m} \leq D_{F l} \leq 2 \times 10^{-5} \mathrm{~m}$ and $20 \mathrm{~m} \geq L_{K} \geq 5 \mathrm{~m}$.

Fig.4 illustrates the role of the additional radial transport coefficients from stochasticity (index DED) in comparison to the "normal" or "classical" values for an exemplary case. Depending on the choice of the stochasticity parameters a strongly increased transport might occur. As to the heat conduction coefficients we note that the classical (anomalous) $\chi_{i}$ and $\chi_{e}$ are related to the diffusion coefficient $\left(\chi_{i} / n_{i}=D_{\perp}, \chi_{e} / n_{e}=3 D_{\perp}\right)$. Fig.4b illustrates the large DED coefficient for electron heat conduction (related to the large classical parallel coefficient). The values increase according to $\zeta_{T}$ leading to locally strongly enhanced energy transport. Fig.4c and Fig.4d compare the classical radial drift of the background plasma ions with additional radial convection from stochasticity, which is always outward directed.
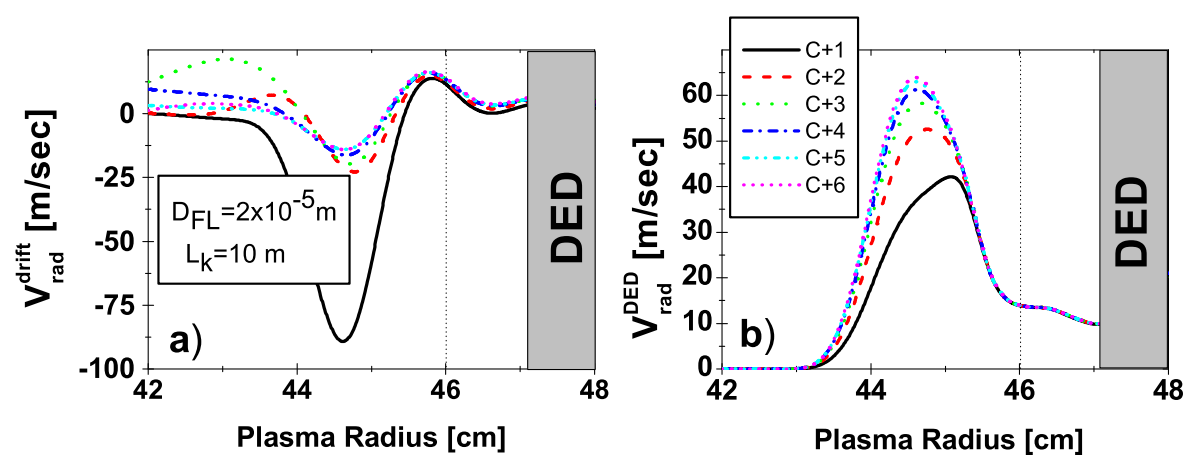

Figure 5: Radial Carbon convection at high-field side: a) carbon ions drift velocities $\left(V_{\text {rad }}^{\text {drift }}\right)$, b) convective velocities due to the stochasticity $\left(V_{r a d}^{D E D}\right)$. Calculations were made for $D_{F L}=2 \times 10^{-5} \mathrm{~m}$ and $L_{K}=10 \mathrm{~m}$. The thin vertical line marks the position of the plasma separatrix.

Fig.5a shows the classical radial drift velocities of the impurities at HFS for case $D_{F L}=$ $2 \times 10^{-5} \mathrm{~m}$ and $L_{K}=10 \mathrm{~m}$, which are usually negative thus favouring transport away from the DED surface. Fig.5b exhibits a dramatic increase of $V_{r z}^{D E D}$ which is the largest when passing from $\mathrm{Z}=1$ to $\mathrm{Z}=6$.

The effect of the stochastic transport increases with $D_{F L}$ and decreases with the Kolmogorov 
length $L_{K}$. For the purpose of our study we have chosen $D_{F L}=2 \times 10^{-5} \mathrm{~m}$ and $L_{K}=10 \mathrm{~m}$, which are used for all the simulations presented in the following, corresponding to relatively strong stochastic transport. This means that the role of stochasticity might be slightly overestimated in the simulations.

\section{Experiment}

The Dynamic Ergodic Divertor in TEXTOR provides an ergodized edge of the plasma in order to control heat and particle exhaust. The sixteen perturbation coils are mounted on the inner side of the tokamak vessel and are parallel to the field lines at $q=3$ [1]. Depending on the current distribution in the coils, a helical magnetic perturbation field with different base mode numbers $\mathrm{m} / \mathrm{n}=3 / 1,6 / 2$ or $12 / 4$ can be created. (the $m$ and $n$ refer to poloidal and toroidal mode numbers). Ergodization is produced by overlapping of perturbations resonant on magnetic flux surfaces with different values of the safety factor q. Together with the formation of a stochastic layer, DED creates magnetic structures at the plasma edge which lead to a local interaction of the plasma with the graphite divertor tiles. On such footprints (see Fig.3) of the magnetic structure deuterium recycling and carbon release might exceed that occurring on the remaining vessel surface up to $50 \%$ in the $12 / 4$ mode. However, since the surface covered by footprints is a negligible fraction of the total interaction surface, the contribution of this increased local production can be disregarded, without loss of generality.

As experimentally shown by the Ergodic Divertor in the tokamak Tore Supra [19] and theoretically predicted [20], at the onset of the perturbing radial field of the DED one might expect an increase of carbon screening and an increase of the ratio of total radiated power to impurity density in the plasma core. On TEXTOR the location of $\mathrm{C}^{+2}$ ions originated from ionization of $\mathrm{C}^{+1}$ is very close to the carbon source both in poloidal and radial directions. For typical TEXTOR discharges the maximum of $\mathrm{C}^{+2}$ ion density is found at about $1 \mathrm{~cm}$ radially inside the plasma column. In contrast, the $\mathrm{C}^{+4}$ ions are nearly uniformly distributed in poloidal direction and are radially located typically at 6-7 $\mathrm{cm}$ inside the plasma. Therefore their distribution is rather insensitive to the position of the carbon source and their density is representative of the carbon content in the confined plasma. The change of the ratio of $\mathrm{C}^{+2}$ line to $\mathrm{C}^{+4}$ line can then be taken as a figure of merit of the change between carbon production and carbon density in the core, i.e. of the change of carbon screening [21].

Measurements of the carbon ions line radiation have been done for different plasma parameters in the TEXTOR-DED tokamk as well as for different currents in the DED coils. In particular, we have analyzed a variety of discharges with different line-integrated densities $\left(1.5 \times 10^{19} \mathrm{~m}^{-3} \leq n_{e} \leq 4.5 \times 10^{19} \mathrm{~m}^{-3}\right)$ and for horizontal shifts of the plasma column in the range of 3 to $5 \mathrm{~cm}$. The input power was in the range $0.35-1.1 \mathrm{MW}$, and the safety factor $\mathrm{q}(\mathrm{a})$ was in the range 2.8 to 3.6 . The currents in the DED coils were also changed $I_{D E D}=7-12$ $\mathrm{kA}$. 


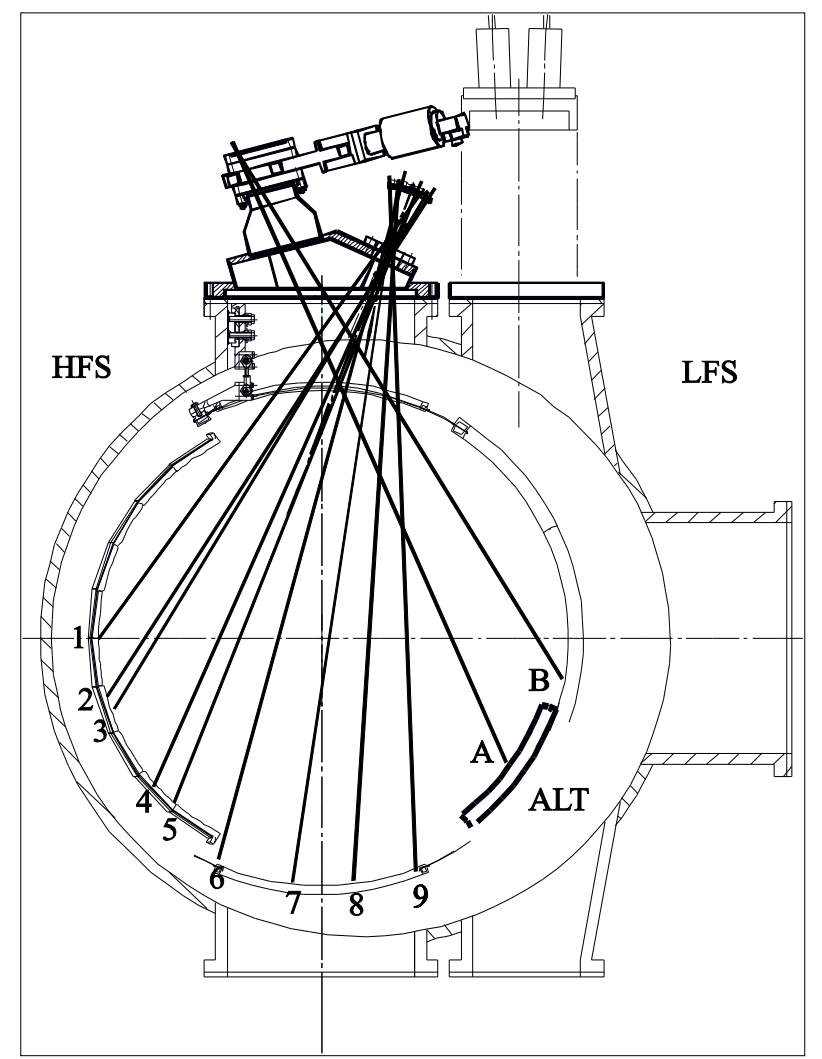

Figure 6: Poloidal cross-section of TEXTOR. Radiation from $\mathrm{C}^{+2}$ and $\mathrm{C}^{+4}$ ions is detected simultaneously along chords 1-9 at a repetition time of 100 msec. Along chords A and B, pointing at the ALT-II toroidal belt limiter, only radiation from $\mathrm{C}^{+2}$ ions is detected.

The following diagnostics were used to study the response of carbon to the application of DED.

\subsection{Diagnostics}

The main diagnostic used for this study involves spectroscopic carbon measurements in the UV range. It is designed to monitor the change of the level of two carbon lines pertaining to $\mathrm{C}^{+2}$ and $\mathrm{C}^{+4}$ ions. In addition bolometric measurements are used to determine the total radiated power, Prad, as well as line integrated bremsstrahlung in the visible for the evaluation of the average ion effective charge, Zeff.

With respect to the carbon diagnostic, the brilliances of the $\mathrm{C}^{+2}$ line at $229.7 \mathrm{~nm}$ and of the $\mathrm{C}^{+4}$ line at $227.1 \mathrm{~nm}$ are detected simultaneously along each of the nine lines of sight ( No 1-9), shown in Fig.6, at a repetition time of 100 ms. Five channels (No 1-5) intercept the divertor plates in the High Field Side where the DED coils are located, the other four (No 6-9) can provide information of carbon behavior far from carbon sources. While for $\mathrm{C}^{+4}$ ions all the nine chords are taken into account, for $\mathrm{C}^{+2}$ ions only radiation emitted from channels 1-5 is considered in this study. This is because the $\mathrm{C}^{+2}$ line radiation arising from chords 6-9 is mainly caused by charge exchange processes, which are not relevant for the estimation of carbon screening. In addition, radiation emitted by the $\mathrm{C}^{+2}$ line in the vicinity of and on the ALT- 
II limiter can be detected (unfortunately, only occasionally for technical reasons) from the two channels A and B in Fig.6. For standard operation of the 12/4 mode the footprints of the magnetic field lines on the divertor targets are located outside the small areas of the divertor intercepted by chords 1-5. Therefore signals for $\mathrm{C}^{+2}$ ions do not include the small contribution given by local interactions at strike zones.

\subsection{Observations}

Since for $12 / 4$ operation of DED the plasma column has to be horizontally shifted towards the divertor tiles in the HFS, we have first compared the intensities of the $\mathrm{C}^{+2}$ line and of the $\mathrm{C}^{+4}$

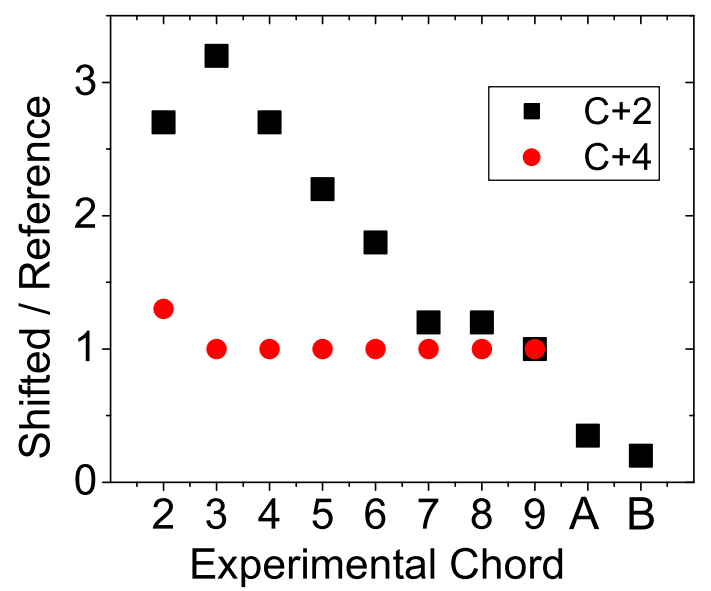

Figure 7: Experimental ratios of carbon line radiation for configurations with and without plasma shift. The shift towards HFS leads to a significant decrease of $\mathrm{C}^{+2}$ line at the LFS and to a simultaneous increase of $\mathrm{C}^{+2}$ at the HFS.

line for the un-shifted (reference) and for the shifted plasma column. In Fig. 7 the ratios of shifted to reference case for $\mathrm{C}^{+2}$ and $\mathrm{C}^{+4}$ are shown for the different channels of the diagnostic (for technical reasons, it was not possible to detect the intensity on chord No. 1 simultaneously with signals from $\mathrm{A}$ and $\mathrm{B}$ ). The brilliance of the $\mathrm{C}^{+4}$ line remains practically unchanged in all channels, while the level of the $\mathrm{C}^{+2}$ line increases up to a factor of three for the channels pointing at the DED target plate and decreases by a factor 3 - 4 for the channels pointing at the LFS around the ALT-II limiter. Comparing the total surface of the target plate with that of the ALT-II limiter (about factor two), one can conclude that the total radiation from $\mathrm{C}^{+2}$ ions does not change significantly by horizontally shifting the plasma, in spite of the significant difference in location. This is in agreement with bolometric data, which show a change within $10 \%$ of Prad when the plasma column is horizontally shifted.

Using the data of channels 1-5 for $\mathrm{C}^{+2}$ and the data of chords 1-9 for $\mathrm{C}^{+4}$ (see Sec.3.1), we have calculated the average values of the level of the $\mathrm{C}^{+2}$ line and of the $\mathrm{C}^{+4}$ line for the reference case (un-shifted), for the shifted case and for the shifted case with DED coil currents switched on (stochastic transport). In Fig. 8a, the ratios of the average $\mathrm{C}^{+2}$ line and of the average $\mathrm{C}^{+4}$ line intensities for the shifted relative to the reference case are shown, together with 

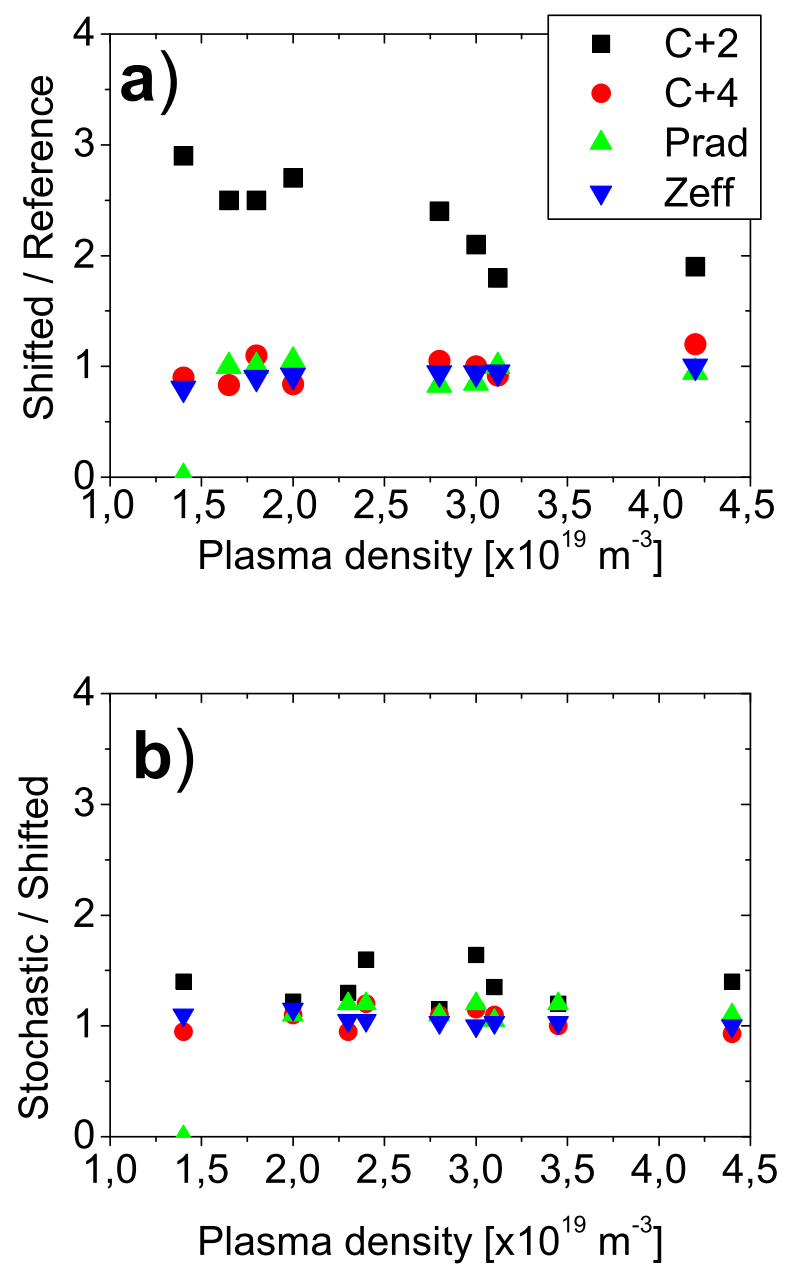

Figure 8: Experimental ratios of radiation, Zeff and carbon ions intensities: a) ratio of shifted case to reference case, b) ratio of stochastic (shifted) case to unperturbed (shifted) case.

the ratios of $Z_{\text {eff }}$ and Prad, for a variety of discharges with different line-integrated densities and for horizontal shifts in the range of 3 to $5 \mathrm{~cm}$. The input power is in the range $0.35-1.1$ MW, and the safety factor $\mathrm{q}(\mathrm{a})$ is in the range 2.8 to 3.6. In Fig. $8 \mathrm{~b}$ the same four ratios are shown for shifted plasmas with DED (stochastic) with respect to shifted without DED current. For the case with DED on, the currents in the DED coils are in the range $I_{D E D}=7-12 \mathrm{kA}$. In both plots the ratios of $\mathrm{C}^{+4}$ line, of Zeff and of Prad are close to one, while a significant difference is seen for the $\mathrm{C}^{+2}$ line. For the shifted to reference case the $\mathrm{C}^{+2}$ line ratio is of the order of 2-3, while for the stochastic to shifted case it is about 1.5. This indicates that the low $\mathrm{Z}$ radiation is more strongly affected by the plasma shift than by the subsequent application of the stochastic transport. In order to identify the physical mechanisms responsible for the observed behavior numerical simulations of plasma transport in the TEXTOR-DED boundary layer have been made. 


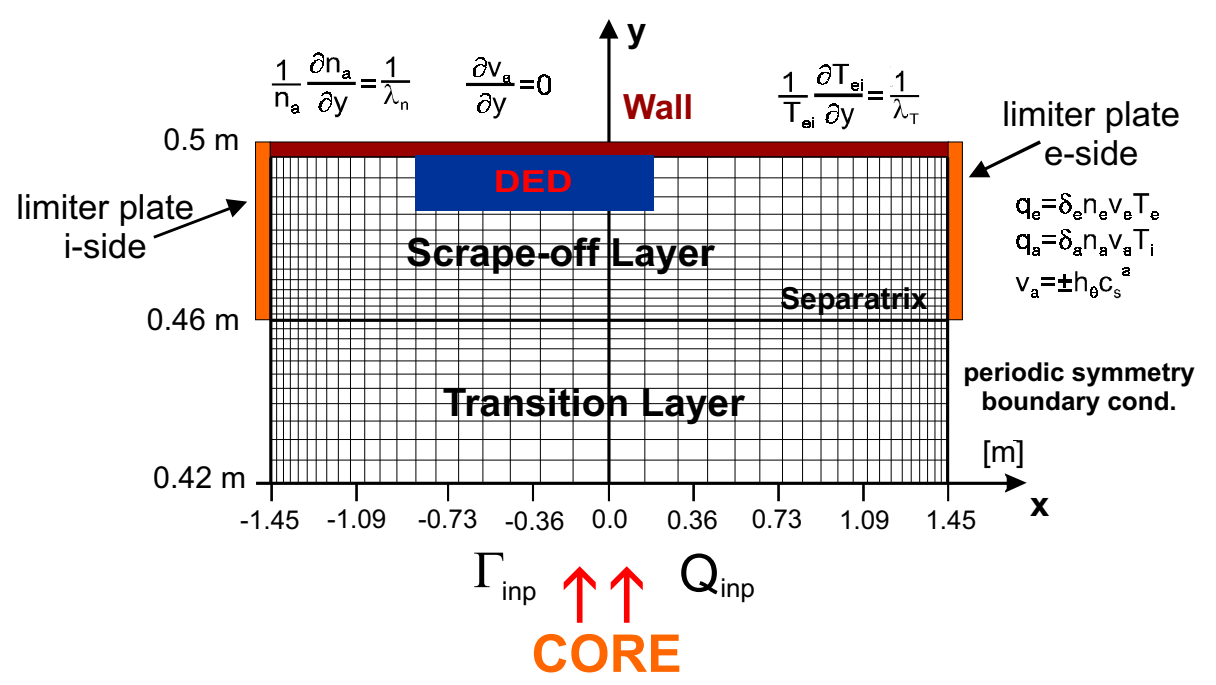

Figure 9: Schematic view of the integration domain with the DED in the shadow of the ALT-II limiter. We show also the boundary conditions used in the TECXY code. The radial interval is always from 0.42 to $0.5 \mathrm{~m}$ and the poloidal distance is from one target side (being ALT-II like in the figure or alternatively DED) to the opposite side.

\section{Simulation results}

We have simulated medium-high density TEXTOR discharges in deuterium with carbon as the dominant intrinsic impurity element. The toroidal magnetic field is $B=2.25 \mathrm{~T}$, the plasma current $I_{p}=350 \mathrm{kA}$ and the Shafranov shift $\triangle=5.5 \mathrm{~cm}$. The DED is introduced into the scrapeoff layer in a region between $-60^{\circ}$ and $+60^{\circ}$ below and above the high-field side. The grid covers the region $42 \mathrm{~cm} \leq r \leq 50 \mathrm{~cm}$, the separatrix is at $a=46 \mathrm{~cm}$, we assume a recycling coefficient $0.7<R<0.85$, and the radial anomalous diffusion coefficient has been assumed $D_{\perp}=1$ or $1.5 \mathrm{~m}^{2} / \mathrm{sec}$. Calculations have been made for the following range of the input particle flux $\left(\Gamma_{i n p}\right)$ and energy flux $\left(Q_{i n p}\right): 2 \times 10^{21} \mathrm{sec}^{-1}<\Gamma_{i n p}<4 \times 10^{21} \mathrm{sec}^{-1}, 0.6 \mathrm{MW}<Q_{\text {inp }}<1 \mathrm{MW}$. In all simulations presented in the following the same level of stochastization corresponding to $D_{F L}=2 \times 10^{-5} m$ and $L_{K}=10 m$ has been taken.

The simulations presented here refer to different geometrical configurations and can be grouped into two different sets. In one case we have simulated the horizontal shift of the plasma column from the ALT-II limiter to the DED target plates, which leads to a displacement of the main plasma sink from $\theta=-45^{\circ}$ to $\theta=180^{\circ}$. Subsequently for the shifted plasmas stochastic transport has been applied. This set of calculations models the global effect of DED operation on TEXTOR in the $12 / 4$ mode.

In the second set of simulations we have assumed that although the main sink is at the ALT-II limiter $\left(\theta=-45^{\circ}\right)$ the DED targets (being not to far from the separatrix) can partly act as additional sink. It should be noted that this configuration has not yet been experimentally investigated in the presence of the edge stochastic field.

In Fig.9 we show a schematic view of our integration domain (together with the boundary conditions used in the TECXY code). The figure directly corresponds to the second set of 


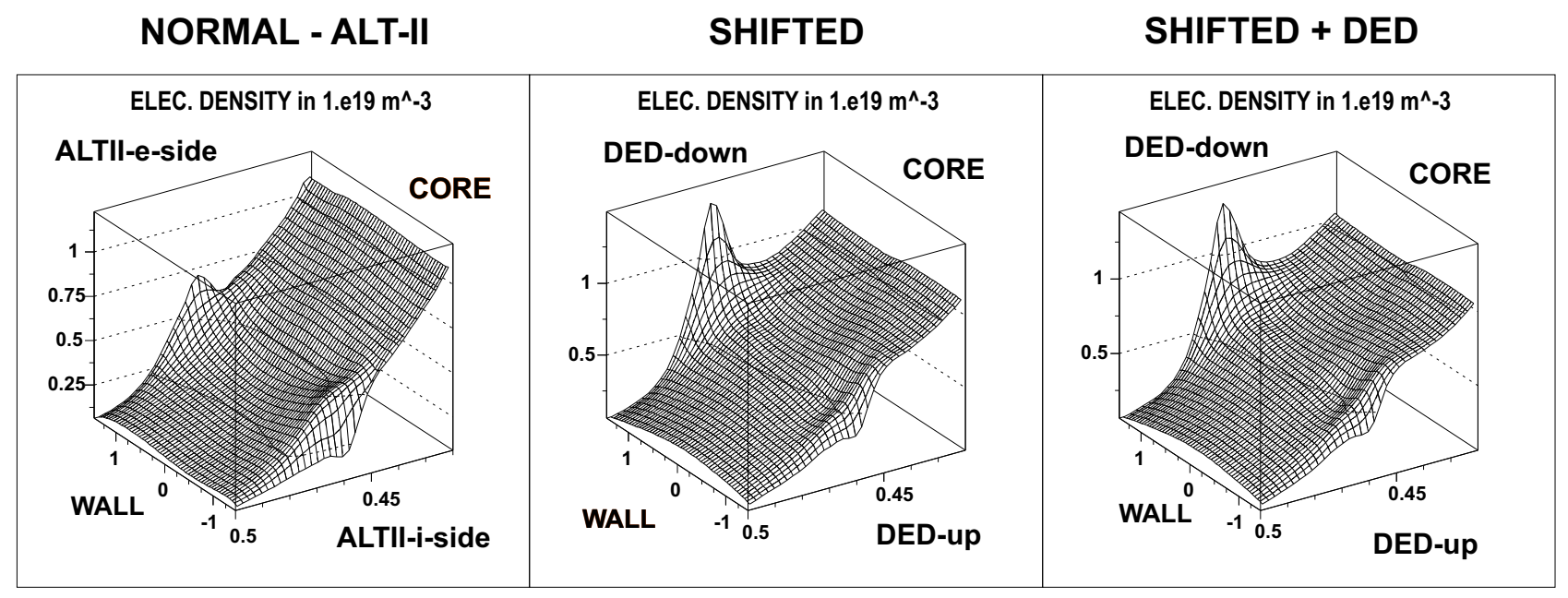

Figure 10: 2D profiles of plasma density. The radial coordinate $(y)$ changes from $0.42-0.5 \mathrm{~m}$ and the poloidal coordinate from one target side (being ALT-II or alternatively DED) to the opposite side $(-1.45 \leq x \leq 1.45)$.

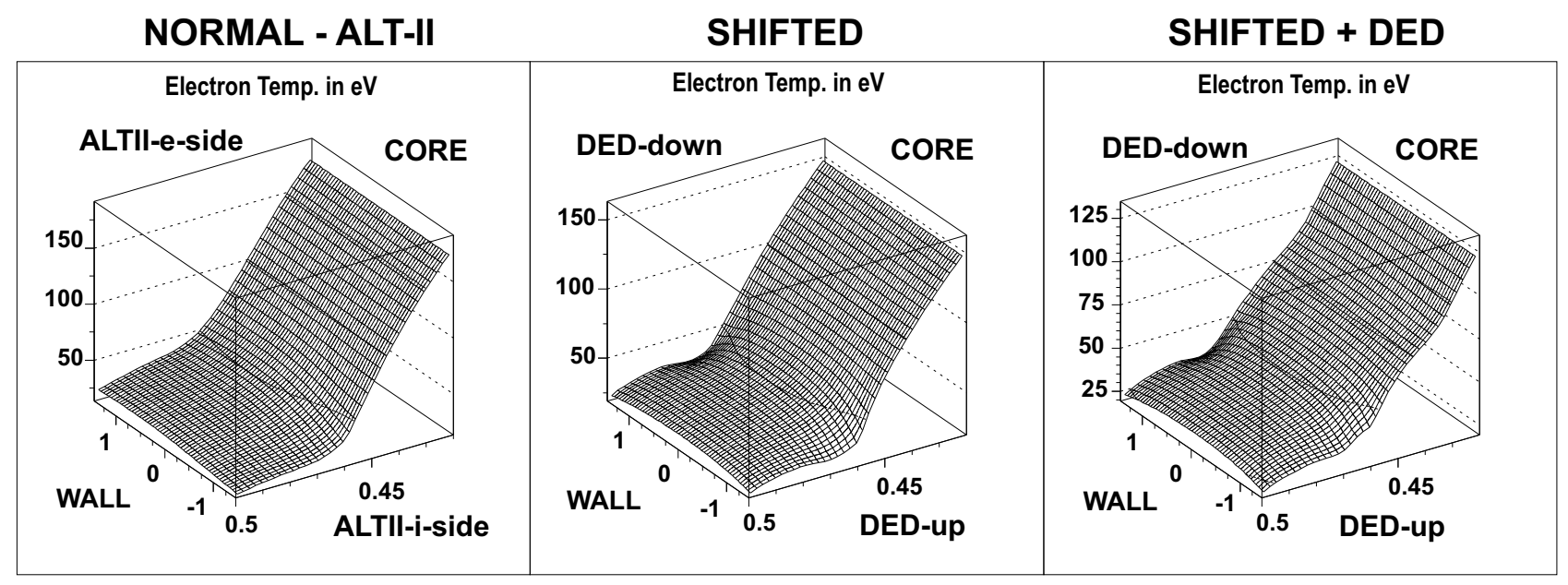

Figure 11: 2D profiles of electron temperature. The radial coordinate $(y)$ changes from $0.42-0.5 \mathrm{~m}$ and the poloidal coordinate from one target side (being ALT-II or alternatively DED) to the opposite side $(-1.45 \leq x \leq 1.45)$.

simulations with the DED targets in the shadow of the ALT-II limiter. We note that the poloidal distance is always from one target side (being ALT-II or alternatively DED) to the opposite side (the poloidal circumference is $2 \times 1.45=2.9 \mathrm{~m}$ ). This means that for the configuration with the main sink at the ALT-II limiter the poloidal angle $\theta$ is in the range $-45^{\circ} \leq \theta \leq 315^{\circ}$ and for the case with the sink at the DED target the range of $\theta$ is $-180^{\circ} \leq \theta \leq 180^{\circ}$.

\subsection{Comparing effects of plasma shift with stochasticity}

In the Figs. 10-13 we show the 2D profiles of electron density, electron temperature and Zeff for three different cases:

a) standard situation with the sink at the ALT-LIMITER $\left(\theta=-45^{\circ}\right)$

b) situation with the sink at the HFS (DED-target) $\left(\theta=180^{\circ}\right)$

c) situation with the sink at the HFS (DED-target) $\left(\theta=180^{\circ}\right)$ and with the stochastic transport 
switched on.

NORMAL - ALT-II

SHIFTED

SHIFTED + DED

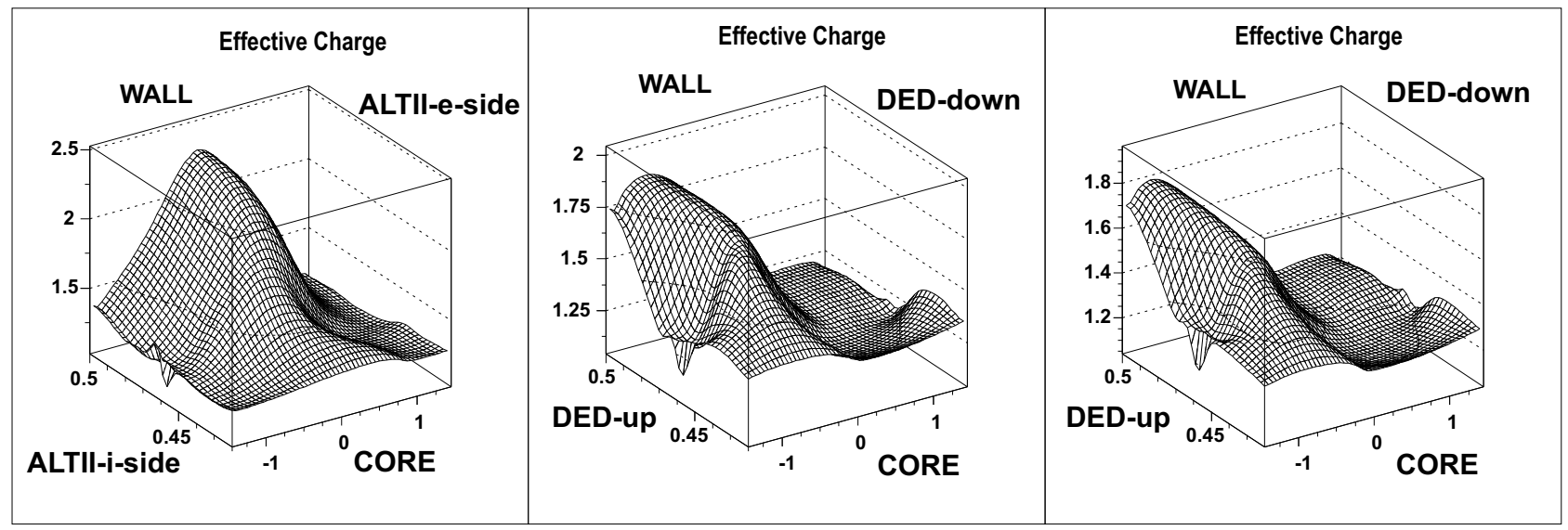

Figure 12: $2 \mathrm{D}$ profiles of effective charge $\left(Z_{\text {eff }}\right)$. The radial coordinate $(y)$ changes from $0.42-0.5 \mathrm{~m}$ and the poloidal coordinate from one target side (being ALT-II or alternatively DED) to the opposite side $(-1.45 \leq x \leq 1.45)$.

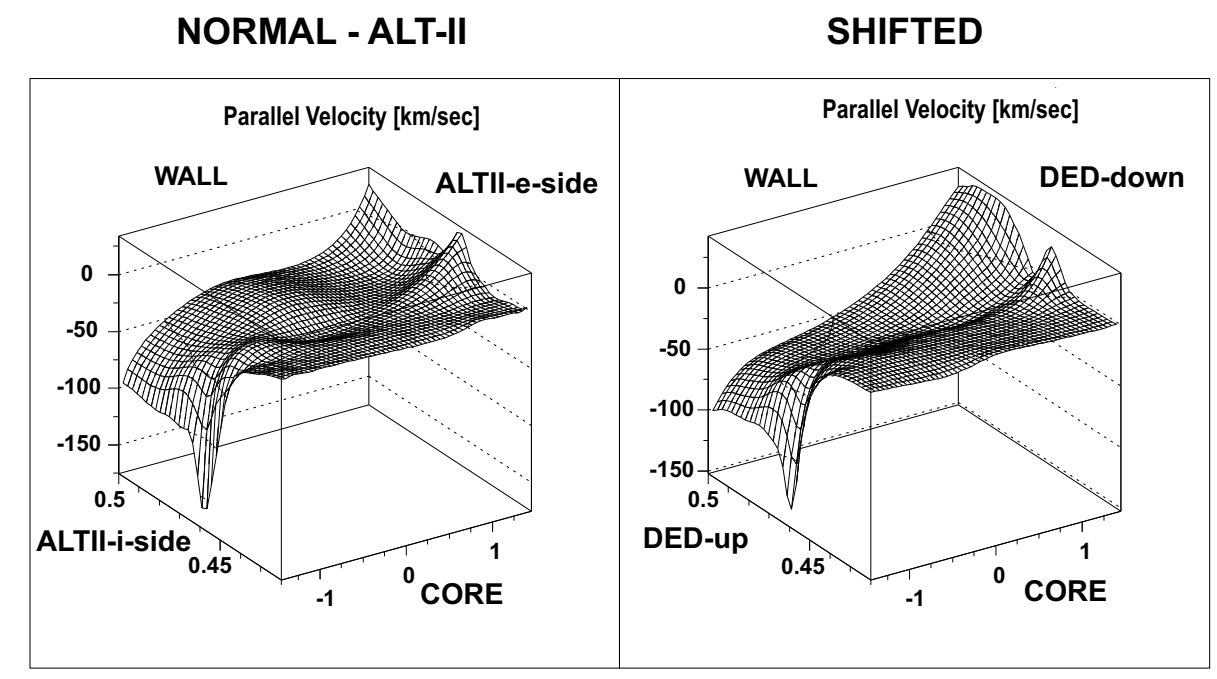

Figure 13: 2D profiles of parallel flow velocity. The radial coordinate $(y)$ changes from $0.42-0.5 \mathrm{~m}$ and the poloidal coordinate from one target side (being ALT-II or alternatively DED) to the opposite side $(-1.45 \leq x \leq 1.45)$.

It can be seen that the change of the position of the sink leads to a strong local peaking of the plasma density in the vicinity of the DED target, and correspondingly to a concomitant drop of the plasma temperature. We note that the steep poloidal gradients developed in front of the target plates lead to conditions of higher plasma recycling. The underlying physical mechanism responsible for these phenomena is associated with the change of the plasma flow pattern in the edge induced by the new position of the sink. Indeed, forces determining the radial electric field are modified and consequently affect the ExB drift and flow distribution [7]). Far from the targets the profiles of the background plasma parameters remain essentially unaffected by the change of the position of the sink. For carbon the effects of plasma shift are more pronounced 
than for the bulk plasma. As seen in Fig.14 the total line radiation (mostly determined by low-Z

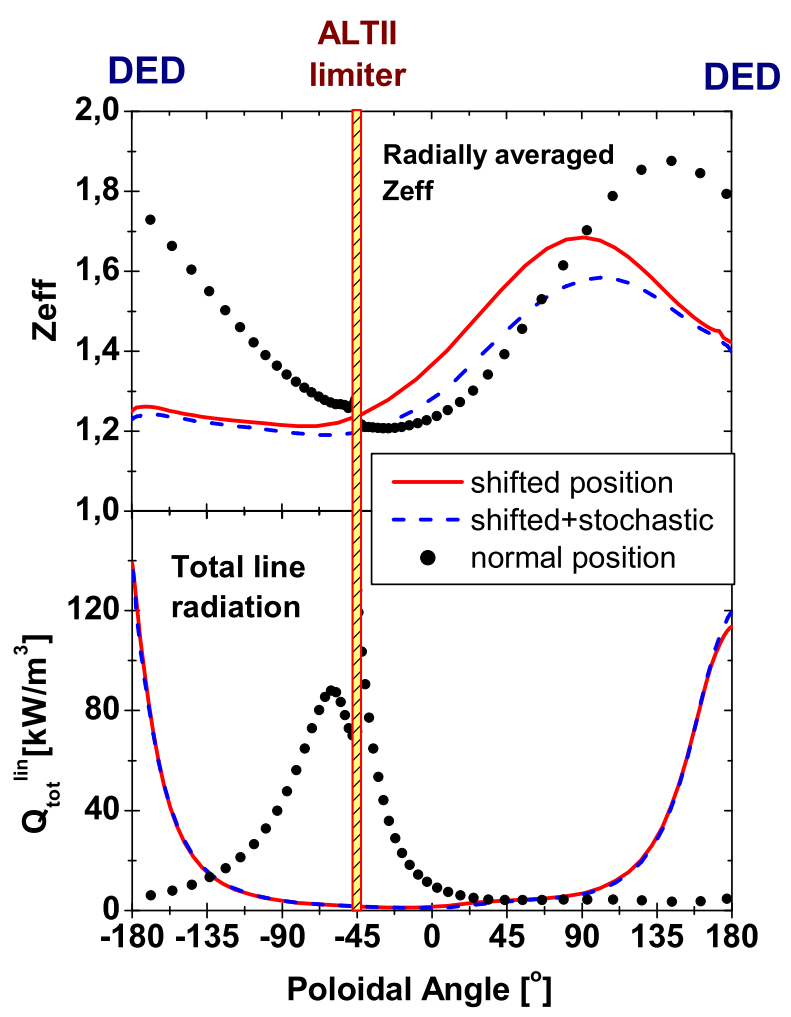

Figure 14: Radially averaged profiles of Zeff and carbon radiation.

carbon ions) increases slightly close to the targets whereas the poloidal position of the radiation is moved from the vicinity of the ALT-II limiter to the HFS region. This can be explained as the combined effect of the change in the position of the carbon source (due to sputtering) together with the increase of plasma density and the corresponding decrease of plasma temperature near the DED target plates. Consequently the ratio between the radiation of the $\mathrm{C}^{+2}$ ions for shifted and not shifted configuration shows, as seen also on the experimental points of Fig.7, a strong increase for poloidal angles close to the DED target (source) and a reduction far away from the DED surface. The behavior of the high-Z ions is reflected by the Zeff profiles which are weakly affected by the plasma shift, consistently with the experimental results for $\mathrm{C}^{+4}$ ions presented in Fig.7. With respect to the high-Z carbon ions the situation is even more complex. It should be noted that in the situation with ALT-II as the main sink the poloidal position of the maximum of $Z_{\text {eff }}$ (representing the position of high- $Z$ ions) is located at the stagnation point (Fig.14) $\left(\theta=135^{\circ}\right)$. However, in the case with the sink at the DED target, the situation is different, since the maximum of $Z_{\text {eff }}$ appears far from the stagnation point and remains more or less at the same poloidal location as for the un-shifted case. These results can be explained by the following sequence. First, we should recall that the position of the maximum of $Z_{\text {eff }}$ is determined by the interplay between thermal and friction forces. In the case with the sink at the HFS the flow pattern in the SOL is strongly changed as can be seen in Fig.13, in which 
parallel velocities in both situations are compared. The increase of parallel flow velocity in the case with the sink at HFS is related to the changes of the poloidal velocities, which are caused by the modification of the radial electric field [7]. As a result, high-Z impurities are shifted in the counterclockwise direction by friction forces from outboard midplane to the upper side of DED.

When the stochastic transport is applied to the shifted plasmas the consequences are rather moderate, as can be seen in Figs. 10-12. The main effect is associated with the reduction of the plasma density and temperature by $10-15 \%$, while the profiles are slightly flattened due to increased radial transport. With regard to impurity ions, the poloidal profile of carbon radiation remains unchanged, and only a small reduction of Zeff is observed (screening efficiency slightly improves, because the friction with the plasma background flow produces a faster outward convection predominantly for high-Z ions). It should be noted that the above presented results are relatively insensitive to changes of the main input parameters of the code such as radial anomalous diffusivity, input energy and particle fluxes as well as plasma recycling.

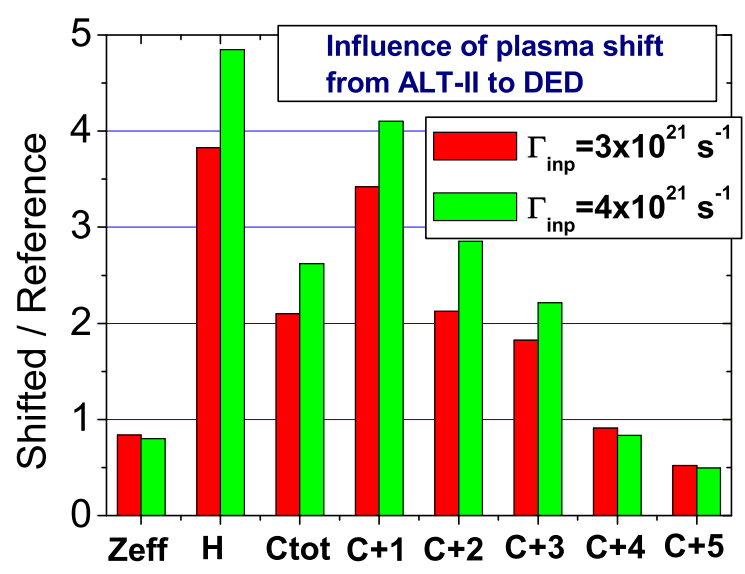

Figure 15: Ratios of Zeff and plasma radiation for shifted and un-shifted plasma for two different input particle fluxes $\Gamma_{i n p}$ (and corresponding densities).

In order to evaluate the global response of some plasma parameters to the plasma position and stochastization we have calculated integrals of Zeff and plasma radiation. The integration is made near the HFS over the poloidal angle corresponding to the region covered by the experimental chords (from top position to the ALT-II limiter, see Fig.6). Radially we have integrated over the full radial width of the plasma boundary considered in our calculations $\left(42 \mathrm{~cm}<r<50 \mathrm{~cm}\right.$ ). In Fig.15, ratios of $Z_{e f f}$ and of the radiation from different species for the shifted to the un-shifted plasma are shown. It can be noted that the radiation of hydrogen and of low-Z ions increases significantly with the plasma shift, for the two considered levels of plasma density. On the other hand the reduction of high- $\mathrm{Z}$ ions density causes only a minor change in Zeff, as in the experimental data reported in Fig.8a.

We should underline, however, that a similar comparison made for the shifted plasma with 
and without stochasticity from DED shows almost no difference between the two cases. These tendencies are again in line with the measurements shown in Fig.8b.

\subsection{DED in the shadow of ALT-II limiter}

So far, with the 3/1-mode of operation of DED, it was impossible to create experimentally a stochastic layer in the edge of a centrally positioned plasma (DED in the shadow of ALT-II limiter) without triggering internal tearing modes. On the other hand, as we have shown, for the 12/4-mode of DED operation stochasticity has only a minor influence on the plasma dynamics as compared with the overwhelming effect of a horizontal plasma shift.

However, it is planned to investigate new operational regimes of TEXTOR-DED (6/2-mode), such that the ergodic layer can be created even in the edge of a plasma which is almost centrally positioned, leading us to predict that in this 6/2-mode case, contrary to the $12 / 4$-mode case, it is the real DED action that controls the plasma dynamics. We point out, that from theory and numerical field line tracing computations for the 6/2-mode configuration, we expect a deeper penetration of the helical perturbation fields, together with a broader zone of stochasticity and about 6-7 times larger $D_{F L}$ values (in agreement with those implemented in our simulation code). This should allow for an important effect of the DED currents on the plasma behaviour without a need for shifting the plasma towards the high field side and DED.

Having this in mind, calculations of the plasma in a configuration with DED in the shadow of ALT-II limiter have been performed. In our simulations we have considered the simultaneous action of two targets: ALT-II being the main limiter, whereas the DED target, located in the scrape-off layer (SOL) near the separatrix, acting as additional sink of particles and energy. It results that in such configuration a very interesting and favourable plasma regime can be developed by switching on stochastic transport.

In Fig.16, the 2D profiles of plasma density and temperature are shown for two situations, without and with stochastic transport. Results have been obtained assuming relatively high plasma density (input particle flux $\Gamma_{i n p}=3 \times 10^{21} \mathrm{sec}^{-1}$ ) with the DED target plates 0.5 $\mathrm{cm}$ away from the separatrix. It can be seen that by switching on stochastic transport the plasma density in the whole transition layer increases significantly. This is accompanied by a corresponding drop of the edge temperature. These phenomena are connected with an increased plasma recycling in the vicinity of the DED target, induced by the stochastic transport. In fact, the enhancement of particle convective flux leads to a nonlinear amplification of the neutral source and recycling. This high recycling zone is broadened radially and extends poloidally up to the i-side of the ALT-II limiter. The strong reduction of the high-Z carbon ion densities seen in Fig.17 indicates a beneficial effect of this regime on impurity screening. Simultaneously, however, the densities of low-Z ions increase due to the stronger source. It should be underlined that this dramatic improvement of the SOL screening efficiency is mainly an indirect effect of the stochastic transport. In fact, differently to the case reported in the previous section (modest screening induced by frictional forces), in this situation the growth of the edge electron density 
is responsible for the reduction of the impurity penetration depth.

However, it should be stressed that this high recycling regime can be created only in a situation with high enough plasma density and when the distance between the DED surface and the separatrix is sufficiently small.

In situations with larger distance between the separatrix and the DED target, the energy flux to the DED surface is reduced, and instead of creating a high density regime a Marfe-like instability develops in front of the DED. Such a situation is similar to the old TEXTOR configuration with bumper limiter at the HFS leading to the development of Marfes for sufficiently high plasma density [11].

In cases with lower plasma density, the distance between separatrix and DED is much less important for the plasma parameters in the edge. Only a weak effect of stochasticity is observed, associated with the increase of the radial transport.

\section{Conclusions}

We have used the 2D multifluid TECXY code in order to simulate some basic features of TEXTOR-DED operation with static fields. In our physical description, the stochasticity in the DED region has been described by a model for optimal paths and locally increased radial transport. The code, despite being very sophisticated and comprehensive, is a two-dimensional approach, hence it can not account for the 3D effects, like for example the laminar zone, island chains or footprint patterns at the DED target plates. Therefore, our calculations do not refer to situations when these effects might be of major importance.

The results of our simulations show that the influence of stochastic transport on plasma in TEXTOR in 12/4-mode operation is relatively small, in spite of the fact that the assumed level of stochasticity is even larger than that applied in the experiment. In most situations the stochastic transport leads only to a reduction of the plasma density and temperature and to a slight flattening of plasma profiles (15-25\% effect). Regarding impurities, a weak improvement of the screening efficiency of high- $Z$ ions is observed. It results from the simulations, that the plasma shift from ALT-II to DED has a stronger effect on the discharge than switching on stochastic transport.

It appears that the calculated tendencies are in line with the experimental observations of the carbon impurity behavior in the TEXTOR-DED discharges. Both the simulation and the experimental evidence indicate that the main changes are related to the horizontal shift of the plasma column, necessary for the penetration of the perturbing $B_{r}$ field, rather than to the stochastic transport itself.

We should stress, however, that for some limited range of plasma parameters, a very favourable regime of plasma operation is predicted to develop when the DED target is slightly in the shadow of the ALT-II limiter. This high density and high-recycling regime is characterized by a substantial increase of the screening efficiency of impurities. 
Finally, it should be noted that the results for the 12/4 mode on TEXTOR-DED differ significantly from the experimental observations on Tore Supra with Ergodic Divertor, where a much stronger effect of the stochastic field on the plasma edge was observed [19], in particular on the impurity screening. Such a better efficiency may arise from the larger perturbation field and shorter Kolmogorov length. Also the geometry might play a role, since the Ergodic Divertor coils on Tore Supra are placed on the LFS, where the magnetic surfaces are more closely spaced than on the HFS, due to the Shafranov shift.

\section{Acknowledgement}

One of the authors (R.Zagórski) has been supported by the grant 3 T10B 00227 from the Polish Committee for Scientific Research and by EC grant G4MA-CT-2002-04037.

The support by the FWO (Fund for Scientific Research-Flanders) is acknowledged.

We thank Dr.Y.Liang for providing us with bolometric data and Dr.M.Jakubowski for valuable discussions on DED magnetic fields.

\section{References}

[1] Finken, K.H. et al., Nucl. Fusion 38 (1998) 515

[2] Gerhauser, H. et al., Nucl. Fus. 42 (2002) 805

[3] Tokar', M.Z., Phys. Plasmas 6 (1999) 2808

[4] Gerhauser, H., Zagórski, R., Claaßen, H.A., Lehnen, M., Contr.Plasma Phys. 40 (2000) 309

[5] Zagórski, R., Reiser, D., Gerhauser, H., Claaßen, H.A., Rapp, J., Contr.Plasma Phys. 40 (2000) 405

[6] Gerhauser, H., Zagórski, R., Claaßen, H.A., Lehnen, M., J.Nucl.Mat. 290-293 (2001) 609

[7] Zagórski, R., Gerhauser, H., Lehnen, M., Loarer, T., Contr. Plasma Phys. 42 (2002) 247

[8] Gerhauser, H., Zagórski, R., Contr. Plasma Phys. 44 (2004) 70

[9] Abdullaev, S.S. et al., Nucl. Fus. 43 (2003) 299

[10] Zagórski, R., Gerhauser, H., Sergienko, G., Contr. Plasma Phys. 44 (2004) 274

[11] Zagórski, R., Gerhauser, H., Physica Scripta 70 Part 2/3 (2004) 173

[12] Zagórski, R., Gerhauser, H., Claaßen, H.A, J. Techn.Physics 40(1) (1999) 99

[13] Zagórski, R. et al., Report of KFA Jülich, JÜL-3829 (Nov.2000)

[14] Gerhauser, H. et al. J.Nucl.Mat., 331-316 (2003) 893

[15] Lehnen M. et al., J.Nucl.Mat. 290-293 (2001) 663 
[16] Jakubowski, M. et al.,"The optimization of the ergodic structure of the dynamic ergodic divertor in the TEXTOR tokamak", to be published in the Proceedings of International Conference PLASMA-2005 on Research and Applications of Plasmas, Opole, Poland, September $6-9,2005$

[17] Abdullaev, S.S. et al., Phys.Plasmas 5(1) (1998) 196

[18] Abdullaev, S.S. et al., Phys.Plasmas 6(1) (1999) 153

[19] Ghendrih, Ph. et al., Plasma Phys. Control. Fusion 38 (1996) 1653

[20] Tokar', M.Z. et al., Plasma Phys. Control. Fusion 39 (1997) 569

[21] Telesca, G. et al., J.Nucl.Mat. 337-339 (2005) 361 


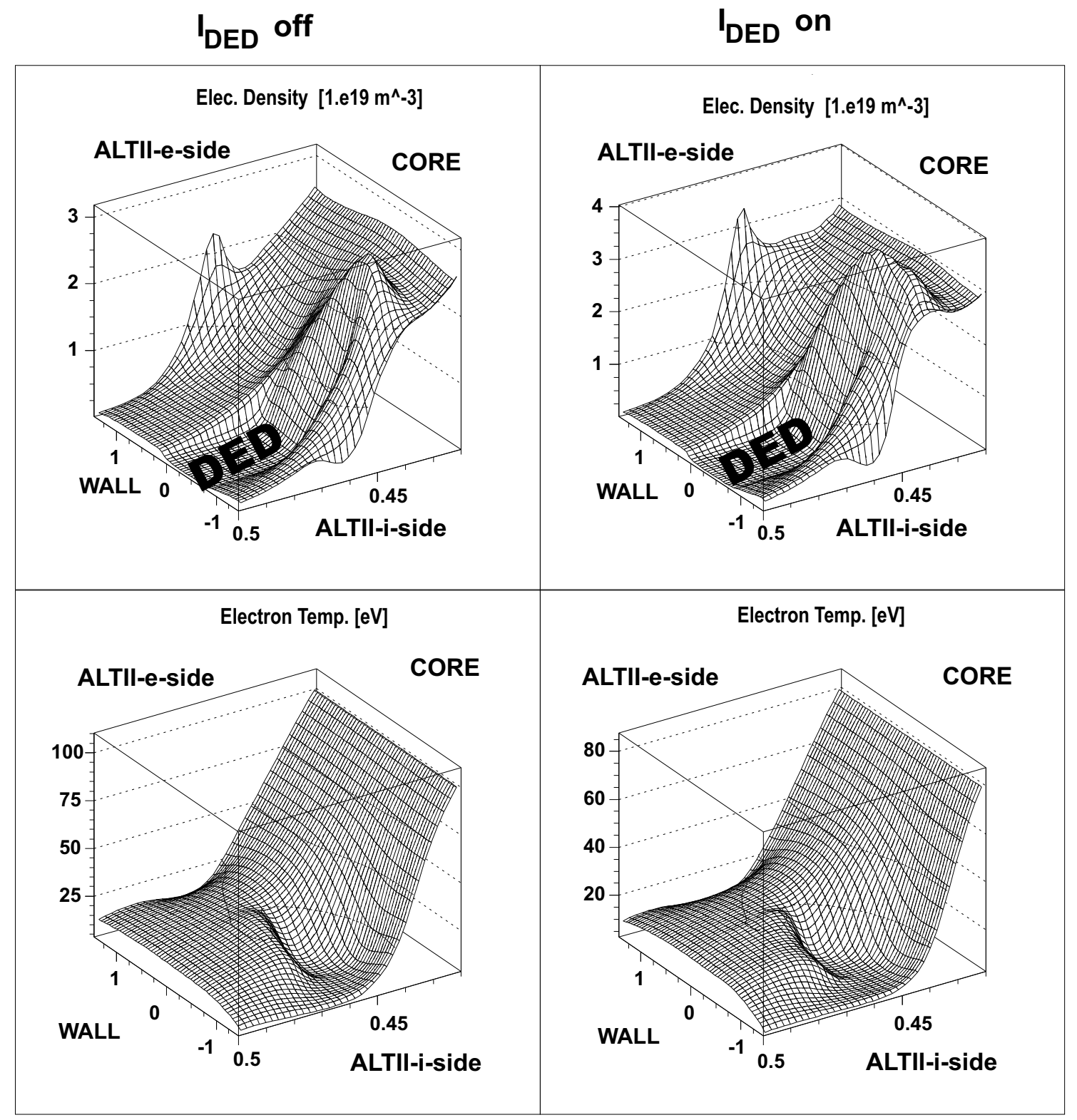

Figure 16: 2D profiles of electron density and temperature. The radial coordinate $(y)$ changes from $0.42-0.5 \mathrm{~m}$ and the poloidal coordinate from i-side of the ALT-II limiter to the opposite e-side $(-1.45 \leq$ $x \leq 1.45)$. 


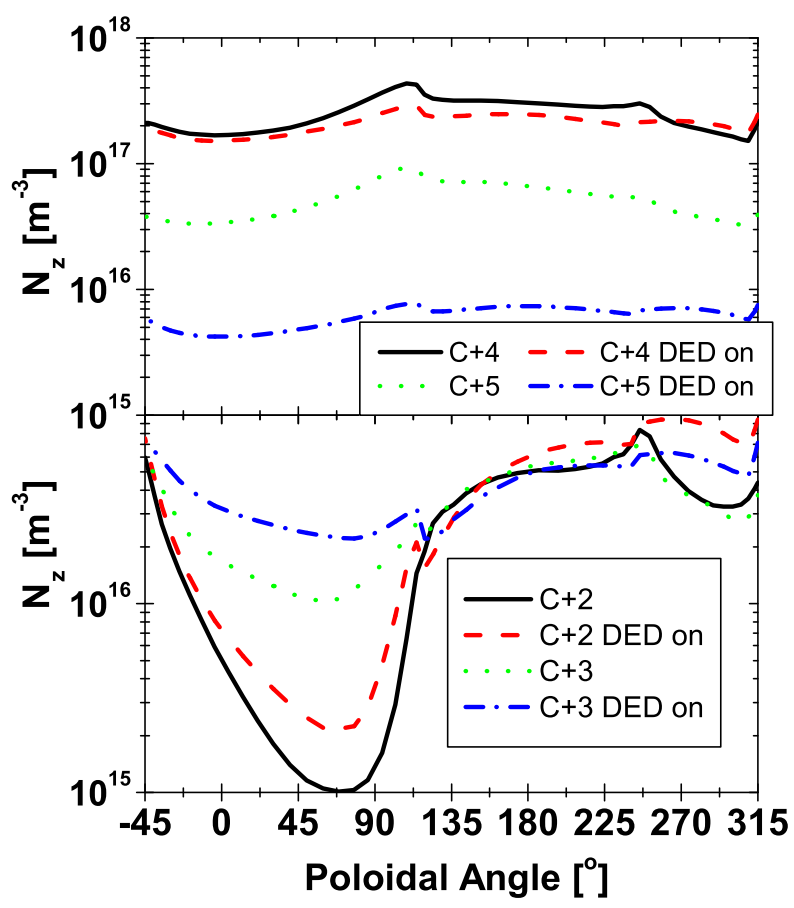

Figure 17: Radially averaged poloidal profiles of carbon ion densities 